Ultrasound-guided aspiration of dominant renal cysts $(>5 \mathrm{~cm})$ followed by injection of absolute ethanol was performed under local anesthesia as an outpatient day procedure. On average, $48.5 \mathrm{ml}$ of sclerosing agent was used per patient (maximum permissible volume $60-70 \mathrm{ml}$ ).

Immediately following the procedure, all patients experienced a burning pain, which usually resolved within 2-4h. Overall, the mean visual analog pain score was significantly lower 7 days after the procedure than at baseline $(P<0.05)$; flank pain persisted in one patient. In total, 48 dominant cysts collapsed and fibrosed following treatment. After 1 year of follow-up, during which time the procedure was repeated in three patients, flank pain and dominant cysts completely disappeared in 13 of the cohort. Mean serum creatinine levels were higher following the procedure and remained so 1 year after treatment $(P<0.001)$, possibly indicating disease progression. One nephrocutaneous fistula and one urinary tract infection were treated successfully; there were no deaths or serious adverse effects.

The authors conclude that alcohol cyst sclerotherapy is a suitable treatment for chronic flank pain in selected patients with ADPKD, but advocate further trials to determine long-term effects on blood pressure and renal function.

Original article Singh I and Mehrotra G (2006) Selective ablation of symptomatic dominant renal cysts using $99 \%$ ethanol in adult polycystic kidney disease. Urology 68: 482-487

\section{C0X2 inhibitors: effect on arrhythmia and renal adverse events}

NSAIDs that block cyclo-oxygenase 1 (COX1 inhibitors), widely used to treat pain and inflammation, are known to have adverse gastrointestinal and renal effects. NSAIDs that are selective for COX2 are associated with a lower risk of adverse gastrointestinal effects, but evidence indicates that they might increase the risk of cardiovascular and renal adverse events. Zhang et al. carried out a meta-analysis to quantify the risk of renal events and arrhythmia associated with COX2 inhibitors.

The meta-analysis included 114 randomized, controlled, double-blind trials of COX2 inhibitors (including rofecoxib, celecoxib, etoricoxib, lumiracoxib, and valdecoxib and parecoxib [data for these two drugs were pooled]), involving 116,094 participants.

Overall, 6,394 renal events (2,670 peripheral edema, 3,489 hypertension, and 235 renal dysfunction) and 286 arrhythmic events occurred. There was significant heterogeneity in the risk of renal events across agents, indicating that a class effect was lacking. Compared with controls, patients taking the first-in-class COX2 inhibitor rofecoxib were at considerably increased risk of renal dysfunction, hypertension, peripheral edema and arrhythmia (relative risks 2.31, 1.55, 1.43 and 2.90, respectively); higher doses ( $>25 \mathrm{mg} /$ day), longer duration of treatment ( $\geq 6$ months) and rheumatoid arthritis further increased the risk of renal events. By contrast, celecoxib-treated patients were not at increased risk of renal events, and there were indications that these patients were at lower risk of renal dysfunction and hypertension than controls (relative risks 0.61 and 0.83 , respectively). Use of other COX2 inhibitors was not significantly associated with an increased risk of adverse renal and arrhythmia events, although further evaluation of etoricoxib and lumiracoxib is warranted.

Original article Zhang J et al. (2006) Adverse effects of cyclooxygenase 2 inhibitors on renal and arrhythmia events: meta-analysis of randomized trials. JAMA 296: 1619-1632 